\title{
Classificação angular para protrusão acetabular utilizada no serviço de ortopedia da Faculdade de Medicina do ABC
}

\author{
Angular classification for protrusio acetabuli used in the orthopedic service of \\ Faculdade de Medicina do ABC
}

Edson Hidenori Miashiro', Takeshi Chikude', Eduardo Nagashigue Yamaguchi', Nelson Keiske Ono', Fausto Boccatto Rosa', Ocilmar Dias do Amaral Junior', Gustavo Martins Fontes', Cléber Furlan', Carlo Milani', Edison Noboru Fujiki'

\begin{abstract}
Resumo
Introdução: a protrusão acetabular foi inicialmente descrita por Otto, em 1824. A incidência é maior no sexo feminino e a bilateralidade, mais comum, é maior nas protrusões de etiologia primária. Sotelo-Garza e Charnley definiram uma classificação que considera a distância da parede medial em relação à linha de Köhler. Objetivo: apresentar a classificação angular para protrusão acetabular e demonstrar sua aplicabilidade. Método: a classificação da Faculdade de Medicina do $A B C$ (FMABC) foi determinada a partir da medida do ângulo $P$ (ângulo de protrusão), formado pela intersecção de três linhas. Foram avaliadas radiografias de bacia em dois grupos de pacientes: o primeiro, com pacientes sem protrusão acetabular, em que foram definidos os valores de normalidade, e o segundo, com pacientes com protrusão acetabular. Destes, foram medidos o ângulo P e o tamanho da protrusão de acordo com Sotelo-Garza e Charnley, sendo os resultados comparados. Dezenove quadris com protrusão foram submetidos à artroplastia total e comparados os valores do ângulo $\mathrm{P}$ no pré e pós-operatório. Resultados: o valor médio do ângulo $P$, em pacientes sem protrusão acetabular, foi $-1,22^{\circ}$. No segundo grupo, observou-se semelhança quando foram comparados os valores da classificação FMABC e os da classificação de Sotelo-Garza e Charnley. A comparação entre os valores do ângulo P pré e pós-operatórios resultou em diferença estatisticamente significante $(p<0,001)$. A classificação angular foi dividida em leve, moderada e grave. Conclusões: 0 ângulo $P$ foi sempre superior a zero nas protrusões acetabulares, os valores numéricos das duas classificações foram estatisticamente significativos, possibilitando a utilização dos mesmos valores da classificação de Sotello-Garza e Charnley para a classificação FMABC, e o uso de enxerto causou uma lateralização do componente acetabular.
\end{abstract}

Palavras-chave: Deformidades articulares adquiridas; acetábulo; artroplastia.

\begin{abstract}
Introduction: protrusio acetabuli was first described by 0tto, in 1824. Its incidence is higher among women and the bilaterality, more common, is higher in primary etiology protrusions. Sotelo-Garza and Charnley set a classification that considers the distance of the medial wall on the Köhler's line. Objective: to present the angular classification for protrusio acetabuli and to demonstrate its applicability. Method: the angular classification of Faculdade de Medicina do ABC (FMABC) was determined from the measure of the $P$ angle (angle of protrusion), formed by the intersection of three lines. Pelvis radiographs were evaluated in two groups of patients: the first, with patients without protrusio acetabuli, in which the values of normality were defined, and the second, with patients who presented the disease. We measured, in the second group, the $\mathrm{P}$ angle and size of the protrusion according to Sotelo-Garza and Charnley, and the results were compared. Nineteen hips with protrusion were submitted to total hip arthroplasty and the values of the $\mathrm{P}$ angle compared in the pre and postoperative. Results: the average value of the $\mathrm{P}$ angle, in patients without protrusio acetabuli, was $-1.22^{\circ}$. In the second group, a similarity was observed when we compared the values of the FMABC angular classification and the Sotelo-Garza and Charnley classification. The comparison between the values of the pre and postoperative $P$ angle resulted in significant statistical difference $(p<0.001)$. The angular classification was divided into mild, moderate and severe. Conclusions: the $P$ angle was always greater than zero in protrusio acetabuli, the values of both classifications were statistically significant, allowing the use of the Sotello-Garza and Chanrley classification values for the FMABC classification, and the use of graft lead to a lateralization of the acetabular component.
\end{abstract}

Keywords: Joint deformities, acquired; acetabulum; arthroplasty.

Recebido: $12 / 8 / 2009$

Revisado: $28 / 11 / 2009$

Aprovado: 16/12/2009

Trabalho realizado no Hospital Estadual Mário Covas da Fundação do ABC, Santo André (SP), Brasil.

' Disciplina de Ortopedia e Traumatologia da Faculdade de Medicina do ABC (FMABC), Santo André (SP), Brasil.

Endereço para correspondência: Edson Hidenori Miashiro - Serviço de Ortopedia do Hospital Mário Covas - Rua Doutor Henrique Calderazzo, 321 -

Paraíso - CEP 09190-615 - Santo André, (SP) - E-mail: edson-miashiro@ig.com.br 


\section{Introdução}

A protrusão acetabular foi inicialmente descrita em 1824 por Adolph William Otto, patologista alemão, por meio de estudos realizados em cadáveres, como uma deformidade da parede medial do acetábulo, com consequente migração da cabeça femoral para o interior da pelve $\mathrm{e}^{\mathrm{l}-\mathrm{6}}$.

As protrusões podem ser idiopáticas (primárias) ou secundárias. Sotelo-Garza e Charnley ${ }^{7}$ observaram, em 182 pacientes, 75,3\% de protrusões primárias. As secundárias podem ser causadas por tumores, infecções, doenças metabólicas, doenças inflamatórias, causas traumáticas ou doenças genéticas ${ }^{1}$. As protrusões acetabulares podem ser obser-

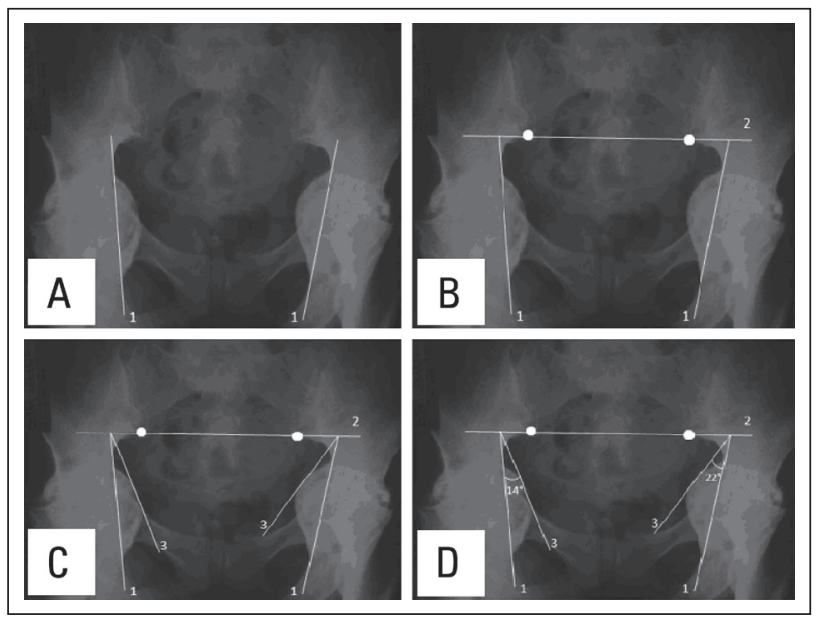

Figura 1 - Radiografia na incidência frente de bacia. (A) linha de Khöler (linha 1); (B) linha formada pela união de dois pontos mais distais das articulações sacroilíacas (linha 2); (C) linha de protrusão (linha 3), que vai do ponto de cruzamento das linhas 1 e 2 até o ponto mais medial da protrusão acetabular; (D) determinação do ângulo de protrusão (ângulo P), formado pela linha de Khöler (linha 1) e a linha de protrusão (linha 3).

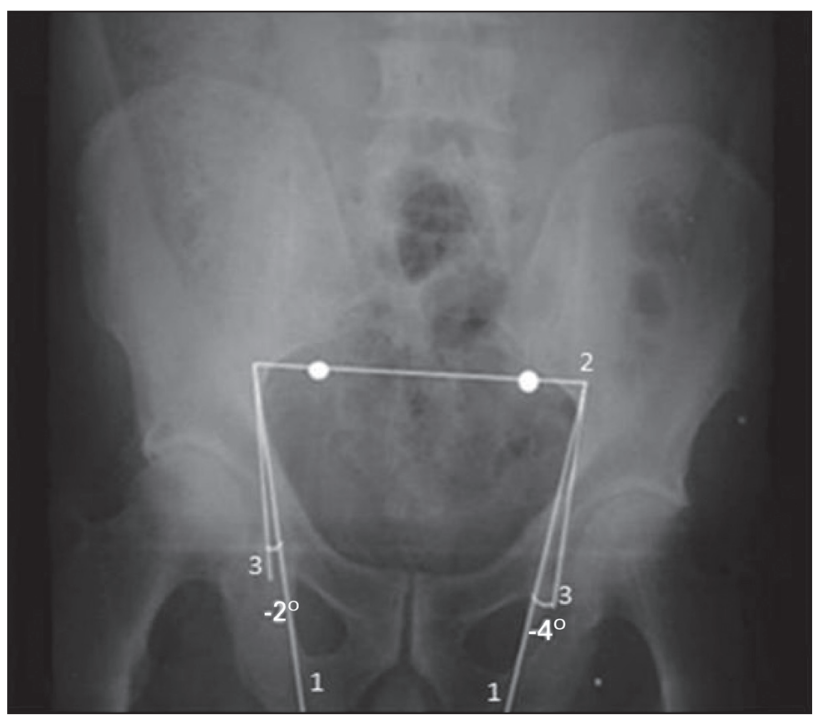

Figura 2 - Radiografia na incidência frente de bacia mostrando 0 ângulo $P$ negativo em paciente sem protrusão acetabular. vadas em $15 \%$ dos pacientes portadores de artrite reumatoide e em 33\% dos pacientes com espondilite anquilosante ${ }^{8,9}$.

A incidência no sexo feminino é maior e da ordem de 10:17. A bilateralidade é mais comum nas protrusões primárias, e as secundárias são mais comumente unilaterais, com exceção das secundárias de artrite reumatoide .

Ranawat et al ${ }^{10}$ sugeriram um método para determinar a correta posição do acetábulo em pacientes com protrusão acetabular. SoteloGarza e Charnley ${ }^{7}$ propuseram uma classificação baseada na distância da parede medial do acetábulo à linha de Köhler, assim como Turibio e Ramos ${ }^{11}$ e Chueire et al. ${ }^{12}$. Desta maneira, a partir da experiência do grupo de cirurgia do quadril da Faculdade de Medicina do ABC (FMABC), objetivou-se descrever classificação para as protrusões acetabulares.

\section{Método}

A classificação da FMABC foi determinada por meio da medida do ângulo de protrusão (ângulo P), medido nas radiografias de bacia na incidência anteroposterior, realizadas conforme padronização feita pelo serviço de Radiologia, baseado nos estudos de Siebenrock et al..$^{13}$

$\mathrm{O}$ ângulo $\mathrm{P}$ foi determinado traçando-se três linhas. A primeira linha é a de Köhler ou linha 1 (Figura 1A); a segunda (linha 2) passa pelo bordo inferior das articulações sacroilíacas, cruzando a linha 1 no ponto V (Figura 1B); a terceira linha, ou linha da protrusão (linha 3), vai do ponto $\mathrm{V}$ até o ponto mais medial da superfície acetabular (Figura 1C). $O$ ângulo $P$ é a medida do ângulo formado pelas linhas 1 e 3 e o ponto V (Figura 1D).

Neste estudo, foram considerados dois grupos. No primeiro, o ângulo $\mathrm{P}$ foi medido em 126 quadris de 63 radiografias de pacientes sem protrusão ou qualquer outra patologia do quadril, com idade variando de 20 a 69 anos, no intuito de se ter um padrão de normalidade (Figura 2). Foi aplicada a análise de correlação de Spearman para avaliar a relação entre o ângulo $\mathrm{P}$ e a idade dos pacientes dentro dessa normalidade. O mesmo foi feito com o teste de Mann-Whitney para observar o valor do ângulo $\mathrm{P}$ e o gênero dos pacientes.

No segundo grupo, foram analisadas radiografias de bacia, na posição anteroposterior, de 21 pacientes com idade variando de 34 a 78 anos, com protrusão acetabular uni ou bilateral, totalizando um total de 34 quadris com protrusão acetabular. Nesses 34 quadris, foi medido o ângulo P, sendo este analisado pela análise de correlação de Spearman, com o intuito de se verificar o grau de relacionamento entre a idade e o valor do ângulo P. Os quadris com protrusão ainda foram classificados segundo Sotelo-Garza e Charnley ${ }^{7}$ Desta forma, os valores numéricos da medida do ângulo P foram comparados estatisticamente com os valores obtidos da classificação de Sotelo-Garza e Charnley, pelo teste dos postos sinalizados de Wilcoxon. No período de agosto de 2005 a agosto de 2008, 16 desses 21 pacientes com protrusão acetabular foram submetidos à artroplastia total de quadril, em um total de 19 quadris 
operados, utilizando-se técnica de artroplastia total cimentada, com enxerto ósseo autólogo de cabeça femoral picado e impactado. Foram usadas próteses Exeter $^{\circledR}$ (Howmedica) e Perfecta ${ }^{\circledR}$ (Whright Medical) (Figura 3A). Nesses quadris operados, foi realizada uma nova medida do ângulo $\mathrm{P}$, no pós-operatório, levando-se em conta na linha da protrusão não o ponto mais medial da protrusão, mas o ponto mais medial da borda do componente acetabular da prótese (Figura 3B). Os novos valores do ângulo $\mathrm{P}$ dos quadris operados foram comparados estatisticamente com o ângulo $\mathrm{P}$ dos quadris pré-operados. Para a aplicação dos testes estatísticos, foi adotado o nível de significância de 5\%.

Esta pesquisa foi aprovada pelo Comitê de Ética da FMABC, sob o parecer $258 / 2007$

\section{Resultados}

No grupo dos 63 pacientes sem protrusão acetabular, 32 (50,79\%) eram do sexo masculino e 31 (49,20\%) do sexo feminino, com idade média de 40,01 anos, variando de 20 a 69 anos. Nestes casos, o ponto mais medial da superfície acetabular foi encontrado lateralmente à linha de Köhler; sendo assim, considerados como tendo valores negativos para o angulo P, ou seja, quando não houver protrusão, o valor do ângulo $\mathrm{P}$ será menor ou igual a zero. O valor médio foi de $-1,22^{\circ}$, variando de $-8^{\circ}$ a $0^{\circ}$. Nesses 126 quadris analisados, o valor mais frequente foi $0^{\circ} \mathrm{em}$ 79 quadris (62,70\%). Foi aplicada a análise de correlação de Spearman, sendo observado que a relação entre o valor do ângulo P e a idade foi estatisticamente não-significante ( $p>0,05)$. Aplicou-se o teste de MannWhitney, observando-se que a diferença entre os gêneros também foi estatisticamente não-significante $(\mathrm{p}>0,05)$.

No grupo dos 21 pacientes com 34 quadris com protrusão acetabular, a idade média foi de 63,09 anos, variando entre 34 e 78 anos. Quanto ao gênero, 19 (90,47\%) eram do sexo feminino e 2 (9,53\%) do sexo masculino. Nesses pacientes, 34 quadris apresentavam protrusão acetabular, sendo 5 com comprometimento unilateral à direita, e 3 à esquerda. Treze pacientes apresentaram protrusão acetabular bilateral. Foram classificados, como protrusões primárias, 29 quadris em 18 pacientes, e como secundárias, 5 quadris em 3 pacientes com diagnóstico de artrite reumatoide. A aplicação da análise de correlação de Spearman, com o intuito de verificar-se o grau de relacionamento entre a idade e o valor do ângulo $\mathrm{P}$ nos quadris com protrusão, mostrou que todos os resultados apresentaram relações estatisticamente não-significantes, podendo-se afirmar que a idade não está relacionada com essas medidas. A aplicação da classificação de Sotelo-Garza e Charnley nos 34 quadris com protrusão acetabular mostrou 6 quadris com protrusões leves, 20 com protrusões moderadas e 8 com protrusões graves. Foi feita a aplicação do teste dos postos sinalizados de Wilcoxon, sendo observado que os valores numéricos obtidos por meio da classificação de Sotelo-Garza e Charnley e da classificação angular foram estatisticamente semelhantes, sendo em 11 quadris exatamente o mesmo valor. A média encontrada, quando utilizada a classificação de Sotelo-Garza e Charnley, foi de 12,17 mm. Quando utilizada a classificação angular, a média foi $11,85^{\circ}$.

A distribuição dos pacientes em relação ao sexo, idade, diagnóstico e valores comparativos das classificações angular e de Sotelo-Garza e Charnley são mostradas no Quadro 1.

Nos 19 quadris operados, foram comparadas as medidas do ângulo $\mathrm{P}$ nos momentos pré e pós-operatório, sendo observado que as medidas foram estatisticamente diferentes, pois $\mathrm{p}<0,001$ (Tabela 1).

Foi observado que a média de diferença entre as medidas do ângulo P no pré e pós-operatório foi de 14,63 (Tabela 2).

Como os valores numéricos obtidos nas duas classificações foram estatisticamente semelhantes, dividimos as protrusões acetabulares, segundo a classificação angular, que denominamos Classificação FMABC para protrusão acetabular, em leve $\left(\operatorname{de~} 1^{\circ}\right.$ a $\left.5^{\circ}\right)$, moderada $\left(\operatorname{de~} 6^{\circ}\right.$ a $\left.15^{\circ}\right)$ e grave (acima de $15^{\circ}$ ). Assim, de acordo com essa divisão, foram encontradas quatro protrusões leves, 20 protrusões moderadas e 10 protrusões graves.

\section{Discussão}

Foram observados, em nossa casuística de 21 pacientes, 18 $(85,71 \%)$ com protrusões primárias e $3(14,28 \%)$ com protrusões secundárias. As protrusões acetabulares puderam ser observadas em 15\% dos pacientes portadores de artrite reumatoide e $33 \%$ dos pacientes com

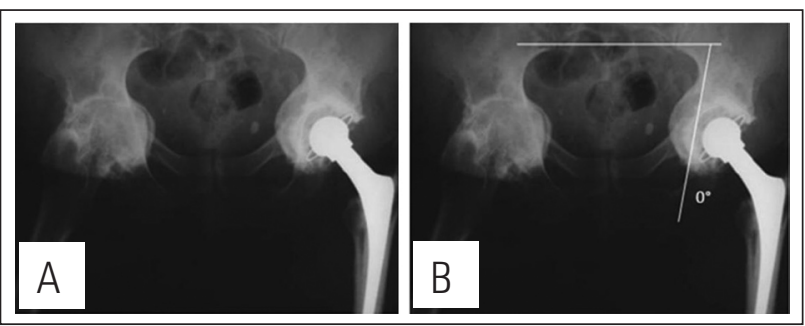

Figura 3 - Radiografia na incidência frente de bacia. (A) Paciente com protrusão acetabular grave submetido a artroplastia total de quadril esquerdo, cimentada com enxerto autólogo de cabeça; $(B)$ Nova medida do ângulo $\mathrm{P}$ em paciente submetido à artroplastia total de quadril esquerdo.

Tabela I - Variáveis do ângulo $\mathrm{P}$ no pré e pós-operatório

\begin{tabular}{lcccccc}
\hline Variáveis & $\mathrm{n}$ & Média & $\begin{array}{c}\text { Desvio } \\
\text { padrão }\end{array}$ & Mínimo & Máximo & $\begin{array}{c}\text { Significância } \\
\text { (p) }\end{array}$ \\
\hline $\begin{array}{l}\text { Ângulo P } \\
\text { pré-operatório }\end{array}$ & 19 & 11,79 & 4,85 & 4 & 22 & \\
$\begin{array}{l}\text { Ângulo P } \\
\text { pós-operatório }\end{array}$ & 19 & $-2,84$ & 3,29 & -8 & 4 & $<0,001$ \\
\hline
\end{tabular}

Tabela II - Diferença entre as medidas do ângulo P no pré e pósoperatório

\begin{tabular}{lccccc}
\hline Variável & $\mathrm{n}$ & Mínimo & Máximo & Média & Desvio padrão \\
\hline $\begin{array}{l}\text { Diferença pré e } \\
\text { pós-operatória }\end{array}$ & 19 & 4 & 28 & 14,63 & 6,22 \\
\hline
\end{tabular}


Quadro I - Pacientes, idade, sexo, diagnóstico e valores comparativos entre as classificações angular e de Sotelo-Garza e Charnley?.

\begin{tabular}{|c|c|c|c|c|c|c|c|}
\hline \multirow[t]{2}{*}{ Nome } & \multirow[t]{2}{*}{ Idade } & \multirow[t]{2}{*}{ Sexo } & \multirow[t]{2}{*}{ Diagnóstico } & \multicolumn{2}{|c|}{ Angular } & \multicolumn{2}{|c|}{ Sotelo-Garza/Charnley } \\
\hline & & & & D & $E$ & D & $E$ \\
\hline $\mathrm{CP}$ & 45 & $\mathrm{~F}$ & Osteoartrite & $4^{\circ}$ & $8^{\circ}$ & $4 \mathrm{~mm}$ & $8 \mathrm{~mm}$ \\
\hline AMSM & 65 & $\mathrm{~F}$ & Osteoartrite & $6^{\circ}$ & $* * * * * * *$ & $5 \mathrm{~mm}$ & $* * * * * * *$ \\
\hline CS & 69 & $\mathrm{~F}$ & Osteoartrite & $16^{\circ}$ & $14^{\circ}$ & $16 \mathrm{~mm}$ & $14 \mathrm{~mm}$ \\
\hline DM & 73 & $\mathrm{~F}$ & Osteoartrite & $16^{\circ}$ & $16^{\circ}$ & $20 \mathrm{~mm}$ & $20 \mathrm{~mm}$ \\
\hline EFA & 46 & $\mathrm{~F}$ & Artrite reumatoide & $14^{\circ}$ & $22^{\circ}$ & $14 \mathrm{~mm}$ & $20 \mathrm{~mm}$ \\
\hline MHS & 49 & $\mathrm{~F}$ & Artrite reumatoide & $4^{\circ}$ & $10^{\circ}$ & $4 \mathrm{~mm}$ & $9 \mathrm{~mm}$ \\
\hline NTA & 74 & $\mathrm{~F}$ & Osteoartrite & $8^{\circ}$ & $* * * * * * *$ & $8 \mathrm{~mm}$ & $* * * * * * * *$ \\
\hline OR & 77 & $\mathrm{~F}$ & Osteoartrite & $12^{\circ}$ & $10^{\circ}$ & $7 \mathrm{~mm}$ & $7 \mathrm{~mm}$ \\
\hline RRC & 56 & $\mathrm{~F}$ & Osteoartrite & $4^{\circ}$ & $4^{\circ}$ & $4 \mathrm{~mm}$ & $4 \mathrm{~mm}$ \\
\hline RRTP & 75 & $\mathrm{~F}$ & Osteoartrite & $14^{\circ}$ & $12^{\circ}$ & $15 \mathrm{~mm}$ & $14 \mathrm{~mm}$ \\
\hline RAF & 77 & $\mathrm{~F}$ & Osteoartrite & $6^{\circ}$ & $16^{\circ}$ & $8 \mathrm{~mm}$ & $14 \mathrm{~mm}$ \\
\hline FCRP & 78 & $\mathrm{~F}$ & Osteoartrite & $* * * * * * *$ & $12^{\circ}$ & $* * * * * * *$ & $14 \mathrm{~mm}$ \\
\hline ILN & 68 & $\mathrm{~F}$ & Osteoartrite & $11^{\circ}$ & $20^{\circ}$ & $14 \mathrm{~mm}$ & $21 \mathrm{~mm}$ \\
\hline ACDG & 34 & $\mathrm{~F}$ & Osteoartrite & $* * * * * * *$ & $10^{\circ}$ & $* * * * * * *$ & $11 \mathrm{~mm}$ \\
\hline ANN & 57 & M & Artrite reumatoide & $25^{\circ}$ & $* * * * * * *$ & $26 \mathrm{~mm}$ & $* * * * * * * *$ \\
\hline MJJ & 68 & $\mathrm{~F}$ & Osteoartrite & $8^{\circ}$ & $10^{\circ}$ & $7 \mathrm{~mm}$ & $11 \mathrm{~mm}$ \\
\hline AMB & 72 & $\mathrm{~F}$ & Osteoartrite & $14^{\circ}$ & $6^{\circ}$ & $10 \mathrm{~mm}$ & $5 \mathrm{~mm}$ \\
\hline WAS & 60 & M & Osteoartrite & $18^{\circ}$ & $* * * * * * * *$ & $15 \mathrm{~mm}$ & $* * * * * * * *$ \\
\hline MLS & 64 & $\mathrm{~F}$ & Osteoartrite & $20^{\circ}$ & $24^{\circ}$ & $16 \mathrm{~mm}$ & $20 \mathrm{~mm}$ \\
\hline MJC & 65 & $\mathrm{~F}$ & Osteoartrite & $* * * * * * *$ & $8^{\circ}$ & $* * * * * * *$ & $8 \mathrm{~mm}$ \\
\hline IMS & 53 & $\mathrm{~F}$ & Osteoartrite & $10^{\circ}$ & $* * * * * * * *$ & $10 \mathrm{~mm}$ & $* * * * * * * *$ \\
\hline
\end{tabular}

espondilite anquilosante ${ }^{8,9}$. Em nossos pacientes, quatro possuíam diagnóstico de artrite reumatoide.

A protrusão acetabular está relacionada com a síndrome de Marfan ${ }^{14-18}$. Kuhlman ${ }^{15}$ observou que, em sua casuística de 22 pacientes com essa síndrome, $45 \%$ apresentavam protrusão acetabular. Yule ${ }^{16}$ observou, em sua casuística de 15 pacientes, $47 \%$ com o mesmo acometimento no acetábulo.

A incidência é maior no sexo feminino, cuja proporção é de 10:17. Em nossos pacientes, foi observada a proporção de 19 (90,47\%) mulheres para $2(9,53 \%)$ homens.

A bilateralidade é mais comum nas protrusões primárias, e as secundárias são mais comumente unilaterais, com exceção das secundárias de artrite reumatoide 7 . Nos 21 pacientes estudados, 3 possuíam o diagnóstico de artrite reumatoide, sendo 2 casos bilaterais. Nas 18 protrusões classificadas como primárias, 7 foram unilaterais, e nas classificadas como secundárias, apenas 1 foi unilateral.

Ranawat ${ }^{10}$ propôs um método para graduar a protrusão acetabular, baseando-se na altura da pelve e sua relação com a altura do acetábulo, a partir de um ponto $5 \mathrm{~mm}$ lateral à intersecção das linhas de Shenton e da linha de Köhler, formando um triangulo isósceles de valor referente a 1/5 da altura da pelve. Sotelo-Garza e Charnley ${ }^{7}$ propuseram uma classificação baseada na distância da parede medial do acetábulo à linha de Köhler, assim como Turibio e Ramos ${ }^{11}$ e Chueire et al. ${ }^{12}$ As duas últimas classificações são medidas em milímetros; portanto, são passíveis de variações causadas pela magnificação observada nas radiografias. É difícil prever a magnificação exata, pois a variação ocorre não apenas pela distância do foco ao filme, mas também pela distância do quadril do paciente ao filme. Os pacientes obesos terão uma magnificação maior ${ }^{15}$. A Classificação FMABC é baseada na mensuração do ângulo P, sendo medida em graus e, portanto, não sofrerá os efeitos da magnificação.

Atualmente, estão sendo usadas as radiografias digitalizadas e até mesmo softwares específicos para o planejamento pré-operatório de $\operatorname{artroplastias~}^{16,17}$. O uso dessas radiografias inviabiliza a utilização das classificações de Sotelo-Garza e Charnley, assim como as de Turibio e Ramos ${ }^{11}$ e Chueire et al. ${ }^{12}$. A Classificação FMABC poderá também ser usada nas radiografias digitais, pois não é medida em milímetros, mas sim em graus.

Harris descreveu o emprego de telas para evitar a migração medial do componente acetabular ${ }^{19}$. Crowninshield ${ }^{20}$, utilizando elementos finitos, observou que, em pacientes com protrusão acetabular, o componente acetabular deve ser colocado em sua posição normal, e não protruso. Nos 19 quadris operados, utilizamos enxerto picado e impactado da cabeça femoral para reforçar a parede medial com próteses totais cimentadas. Têm sido descritos bons resultados com o uso de enxerto impactado nos quadris com protrusão acetabular ${ }^{21-27}$. Hirst ${ }^{21}$ observou que o enxerto funcionou como um espaçador, permitindo a lateralização do componente acetabular, protegendo a fina parede medial dos efeitos térmicos da polimeralização do cimento ósseo.

Nos 19 quadris operados, foram comparadas as medidas do ângulo $\mathrm{P}$ nos momentos pré e pós-operatório, sendo observado que as medidas foram estatisticamente diferentes, com $\mathrm{p}<0,001$. Portanto, foi demonstrado que o enxerto causou a lateralização do componente acetabular em todos os casos. Observou-se que a média de diferença entre as medidas do ângulo P no pré e pós-operatório foi de 14,63․ Entre- 
tanto, nossos casos são recentes e o acompanhamento pós-operatório não nos permite maiores conclusões com relação à migração medial progressiva do componente.

Outros autores relataram bons resultados com o uso de componentes acetabulares não cimentados ${ }^{28,29}$.

Os 34 quadris foram medidos segundo a Classificação FMABC e a classificação de Sotelo-Garza e Charnley, e os valores numéricos obtidos foram comparados. Foi feita a aplicação do teste dos postos sinalizados de Wilcoxon, sendo observado que os valores numéricos obtidos nas duas classificações foram estatisticamente semelhantes. Devido à grande semelhança entre ambas as classificações, sugerimos o uso dos mesmos valores numéricos de Sotelo-Garza e Charnley, porém com medidas em graus. Desta forma, propõe-se que a protrusão será leve, de $1^{\circ}$ a $5^{\circ}$, moderada, de $6^{\circ}$ a $15^{\circ}$, e grave, acima de $15^{\circ}$.
A Classificação FMABC é uma forma de aplicar a classificação de Sotelo-Garza e Charnley e poderia ser utilizada nas radiografias digitais.

Em conclusão, o ângulo $\mathrm{P}$ em pacientes com protrusão acetabular é sempre maior que zero graus; houve predominância da protrusão acetabular em $90 \%$ dos pacientes do gênero feminino, e os valores numéricos entre as duas classificações foram estatisticamente significativos, possibilitando a utilização dos mesmos valores da classificação de Sotello-Garza e Charnley para a Classificação FMABC. Não existe correlação entre o aumento da idade e o aumento do grau da protrusão acetabular. As protrusões acetabulares foram classificadas, segundo a Classificação FMABC, em leve $\left(1^{\circ}\right.$ a $\left.5^{\circ}\right)$, moderada $\left(6^{\circ}\right.$ a $\left.15^{\circ}\right)$ e grave (acima de $15^{\circ}$ ). O uso do enxerto de cabeça femoral impactado no acetábulo permitiu a lateralização do componente acetabular com consequente diminuição do grau da protrusão acetabular.

\section{Referências}

1. Van de Velde $S$, Fillman $R$, Yandow $S$. The aetiology of protrusio acetabuli. Literature review from 1824 to 2006. Acta Orthop Belg. 2006;72(5): $524-9$.

2. McBride MT, Muldoon MP, Santore RF, Trousdale RT, Wenger DR. Protrusio acetabuli: diagnosis and treatement. J Am Acad Orthop Surg. 2001; 9(2):79-88.

3. Dunlop CCR, Jones CW, Maffulli N. Protrusio acetabuli. Bulletin Hospital for Joint Diseases. 2005;65(3/4):105-14.

4. Alexander $\mathrm{C}$. The aetiology of primary protrusio acetabuli. $\mathrm{Br} \mathrm{J}$ Radiol. 1965;38:567-80

5. Hooper JC, Jones EW. Primary protrusion of the acetabulum. J Bone Joint Surg Br. 1971;53(1):23-9

6. Simmons EH, Gibson DA. Otto pelvis associated with familial degenerative arthrosis of the hip joint. Can J Surg. 1966;9(3):268-72.

7. Sotelo-Garza A, Charnley J. The results of Charnley arthroplasty of the hip performed for protrusio acetabuli. Clin Orthop Relat Res. 1978; 132:12-8

8. Hastings DE, Parker SM. Protrusio acetabuli in rheumatoid arthritis. Clin Orthop Relat Res. 1975;(108):76-83.

9. Dwosh IL, Resnick D, Becker MA. Hip involvement in ankylosing spondylitis. Arthritis Rheum. 1976;19(4):683-92.

10. Ranawat CS, Dorr LD, Inglis AE. Total hip arthroplasty in protrusio acetabuli of rheumathoid arthritis. J Bone Joint Surg Am. 1980;62(7):1059-65.

11. Turibio FM, Ramos WM. Protrusão acetabular do quadril. In: Turibio FM. Manual de patologias do quadril adulto. São Paulo: Escola Paulista de Medicina; 1993. p. 49-51.

12. Chueire AG, Rejaili WA, Santos AF. Protrusão acetabular (Otopelve). Acta Ortop Bras. 2002;10:52-7.
13. Siebenrock KA, Kalbermatten DF, Ganz R. Effect of pelvic tilt on acetabular retroversion: a study of pelves from cadavers. Clin Orthop Relat Res. 2003;(407):241-8

14. Van de Velde S, Fillman R, Yandow S. Protrusio acetabuli in Marfan syndrome. History, diagnosis, and treatment. J Bone Joint Surg. 2006;88(3):639-46.

15. Kuhlman JE, Scott WW Jr, Fishman EK, Pyeritz RE, Siegelman SS. Acetabular protrusion in the Marfan syndrome. Radiology. 1987;164(2):415-7.

16. Yule SR, Hobson EE, Dean JC, Gilbert FJ. Protrusio acetabuli in Marfan's syndrome. Clin Radiol. 1999;54(2):95-7.

17. Do T, Giampietro PF, Burke SW, Davis JG, Raggio C, Schneider R, et al. The incidence of protrusio acetabuli in Marfan's syndrome and its relationship to bone mineral density. J Pediatr Orthop. 2000;20(6):718-21.

18. Sponseller PD, Jones KB, Ahn NU, Erkula G, Foran JR, Dietz HC 3rd. Protrusio acetabuli in Marfan syndrome: age-related prevalence and associated hip function. J Bone Joint Surg Am. 2006;88(3):486-95.

19. Harris $\mathbf{W H}$, Jones WN. The use of wire mesh in total hip replacement surgery. Clin Orthop Relat Res. 1975;(106):117-21.

20. Crowninshield RD, Brand RA, Pedersen DR. A stress analysis of acetabular reconstruction in protrusio acetabuli. J Bone Joint Surg Am. 1983;65(4):495-9.

21. Hirst P, Esser M, Murphy JC, Hardinge K. Bone grafting for protrusio acetabuli during total hip replacement. A review of the Wrightington method in 61 hips. J Bone Joint Surg Br. 1987;69(2):229-33.

22. Kinzinger PJ, Karthaus RP, Slooff TJ. Bone grafting for acetabular protrusion in hip arthroplasty. 27 cases of rheumatoid arthritis followed for 2-8 years. Acta Orthop Scand. 1991;62(2):110-2.

23. Mibe J, Imakiire A, Watanabe T, Fujie T. Results of total hip arthroplasty with bone graft and support ring for protrusio acetabuli in rheumathoid arthritis. $J$ Orthop Sci. 2005;10(1):8-14. 
24. Schreurs BW, van Tienen TG, Buma P, Verdonschot N, Gardeniers JW, Slooff TJ. Favorable results of acetabular reconstruction with impacted morsellized bone grafts in patients younger than 50 years: a 10- to 18-year follow-up study of 34 cemented total hip arthroplasties. Acta Orthop Scand. 2001;72(2):120-6.

25. McCollum DE, Nunley JA, Harrelson JM. Bone-grafting in total hip replacement for acetabular protrusion. J Bone Joint Surg Am. 1980;62(7):1065-73.

26. Rosenberg WW, Schreurs BW, de Waal Malefijt MC, Veth RP, Slooff TJ. Impacted morsellized bone grafting and cemented primary total hip arthroplasty for acetabular protrusion in patients with rheumatoid arthritis: an 8- to 18-year follow-up study of 36 hips. Acta Orthop Scand. 2000;71(2):143-6.

27. Mayer G, Hartseil K. Hip replacement in acetabular protrusion. Acta Orthop Scand. 1985;56(6):461-3.

28. Sharp DJ, Porter KM, Duke RF. The resolution of protrusio acetabuli treated with Ring's hip prosthesis. J Bone Joint Surg Br. 1984;66(5):635-8.

29. Matsuno H, Yasuda T, Yudoh K, Yonezawa T, Nakazawa F, Murata T, et al. Cementless cup supporter for protrusio acetabuli in patients with rheumatoid arthritis. Int Orthop. 2000;24(1):15-8. 